\title{
FREQUENCY AND ASSOCIATION OF SYMPTOMS DUE TO CONTACT WITH A SUSPECTED OR CONFIRMED CASES OF COVID IN RHEUMATOLOGIC PATIENTS
}

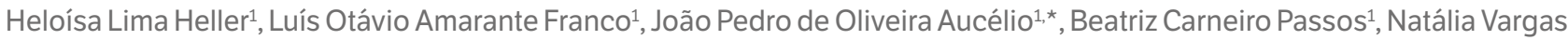
do Nascimento ${ }^{2}$, Lorenna Alves Bezerra ${ }^{1}$, Julia Dourado Paiva ${ }^{1}$, Mariana Oliveira Santana ${ }^{1}$, Maria Clara Potiguara Azevedo Teixeira ${ }^{1}$, Esther Cabral Jersey ${ }^{1}$, Beatriz Pires Paes ${ }^{1}$, Marcos Igor Albanaz Vargas ${ }^{1}$, Gabriela Furlan Ribeiro Barbosa Netto ${ }^{2}$, Gabriela Brill Ney ${ }^{3}$, Viviane Cristina Uliana Peterle1, Ana Paula Gomides ${ }^{1}$

1.Centro Universitário de Brasília, Brasília (DF), Brazil; 2.Universidade Católica de Brasília, Brasília (DF), Brazil; 3.Centro Universitário do Planalto Central Apparecido dos Santos, Brasília (DF), Brazil.

*Corresponding author: jp.aucelio@gmail.com

\section{BACKGROUND}

Severe acute respiratory syndrome coronavirus 2 (SARS-CoV-2) is an acute respiratory disease, responsible for a severe global health crisis. A suspected case is considered in patients who manifest flu-like symptoms and were in contact with a confirmed case. A confirmed case is defined after serological or molecular laboratory confirmation. The progression of the disease is associated with an inflammatory condition, thus raising the hypothesis that rheumatologic patients in chronic use of antimalarials or immunosuppressants could present lower rates of moderate to severe COVID-19. Additionally, corticosteroids such as dexamethasone and methylprednisolone improved dyspnea and cough. The present study relates rheumatologic patients in use of immunosuppressants, antimalarials and/or corticosteroids and the development of symptoms of COVID-19 after contact with a suspected or confirmed case of COVID-19.

\section{METHODS}

This study was part of the Mario Pinotti study. A telephone-based survey was carried out to evaluate residents of the Federal District with rheumatic diseases. After the consent of the participants, data was collected on the RedCap platform. The interviews occurred in an interval of 14 days, totaling 5 contacts in the period from April to June 2020. The collected data was analyzed using descriptive statistics. As for frequency of flu/virus symptoms, their summary measures by characteristics were based on the Mann-Whitney or Kruskal-Wallis test. The correlation with the characteristics were performed by the Spearman correlation.

\section{RESULTS}

Mean frequencies of symptoms per contact with a suspected/confirmed case of coronavirus infection $(p=0.005)$ and use of immunosuppressants $(p<0.001)$ were divergent. There is a higher frequency of symptoms in patients who had contact with suspected/confirmed cases in comparison to those who had no contact. In addition, those who did not use immunosuppressants had a lower mean frequency of symptoms than those who used this drug. There was also a weak negative association between age and the frequency of symptoms ( $r S=-0.211, p<0.001$ ), indicating that the higher the age, the lower the frequency of flu or virus symptoms. The correlation between the frequency of symptoms, weight and duration of antimalarial use was not significant.

\section{CONCLUSION}

Data on the frequency of symptoms of COVID-19 in rheumatologic patients, after contact with suspected or confirmed cases, are divergent and do not show a considerable positive or negative relationship. However, there is a higher frequency of symptoms in immunosuppressed individuals when compared to the general population.

\section{KEYWORDS}

Flu-like symptoms, Rheumatologic patients, COVID-19. 